\title{
A CASE REPORT OF GRANULOMATOUS ROSACEA of the FACE
}

Singapore Med J 2018; 59(4): 228-229 https://doi.org/10.11622/smedj.2018047

Dear Sir,

Rosacea is a chronic and relapsing inflammatory skin disorder that occurs in adults over 30 years of age, with a female preponderance. Rosacea has a varied clinical presentation, including congestion, flushing, telangiectasia and rhinophyma. Although there are four subtypes, ${ }^{(1)}$ clinical overlap of the features may present in the same patient.

Our patient was a 61-year-old Chinese woman who presented with an acute onset of facial rashes for two weeks, without any prior triggers. Physical examination revealed extensive erythematous papules that coalesced into plaques over the cheeks, nose, glabella and chin (Fig. 1a). No ocular involvement or telangiectasia was noted. She was initially treated with oral tetracycline $250 \mathrm{mg}$ twice daily and topical metronidazole for two months, but there was no improvement. Her condition worsened and she was treated for rosacea fulminans with oral prednisolone therapy at $0.5 \mathrm{mg} / \mathrm{kg} /$ day and oral doxycycline $100 \mathrm{mg}$ twice daily. A skin biopsy was performed and the histology report revealed parakeratosis with acanthosis and spongiosis with exocytosis of lymphocytes. Within the dermis, non-caseating granulomatous inflammation, composed of lymphocytes, epithelioid histiocytes and some foreign bodytype multinucleated giant cells, was seen. The Ziehl-Neelsen stain for acid-fast bacilli on histology was negative and the anti-nuclear antibody screen was also normal. A diagnosis of granulomatous rosacea (GR) was made. The oral steroids were gradually tapered over eight weeks and the patient showed significant clinical improvement (Fig. 1b). Due to persistent erythema, daily topical brimonidine was added with good progress. Oral doxycycline was continued for six months and she remained in remission.

Differential diagnoses include GR, acne agminata, perioral dermatitis and micropapular sarcoidosis. Clinically, GR appears to be a distinct form of rosacea. Patients present with firm, yellow, red, brown, or flesh-coloured papules or nodules localised around the eyes, nose, and mouth on relatively normal-appearing skin.(1) The pathognomonic feature of GR on histology is the formation of noncaseating granulomas in the superficial and mid dermis, which can manifest as a large, central empty space or may be small palisaded, elastolytic or diffuse. ${ }^{(2)}$ Acne agminata, on the other hand, presents with a predilection for the periorbital region, which is not normally seen in rosacea, and the histologic finding of caseating granulomas is key. ${ }^{(3)}$ Perioral dermatitis, however, is seen in younger women and children, and resembles rosacea clinically and histologically. Patients may present with confluent erythematous papules, papulovesicles and papulopustules of the face with characteristic sparing of the vermilion border. ${ }^{(4)}$ Micropapular sarcoidosis may present at any age with facial papules and accompanying systemic symptoms of fatigue, weight loss and joint pain, as well as pulmonary symptoms.

There is no standard treatment for GR. Oral antibiotics such as tetracycline or doxycycline are usually used. Other therapeutic modalities include topical azelaic acid, benzoyl peroxide, metronidazole, corticosteroids, pimecrolimus and systemic corticosteroids. ${ }^{(5)}$ For recalcitrant GR, isotretinoin treatment may be attempted. Rosacea treatment is tailored to the subtype and phenotypic features displayed. ${ }^{(6)}$ Brimonidine gel, an alpha-adrenergic vasoconstrictor, is a newly approved treatment for erythema in rosacea and was prescribed to treat our patient's residual baseline flushing after resolution of the granulomatous component.
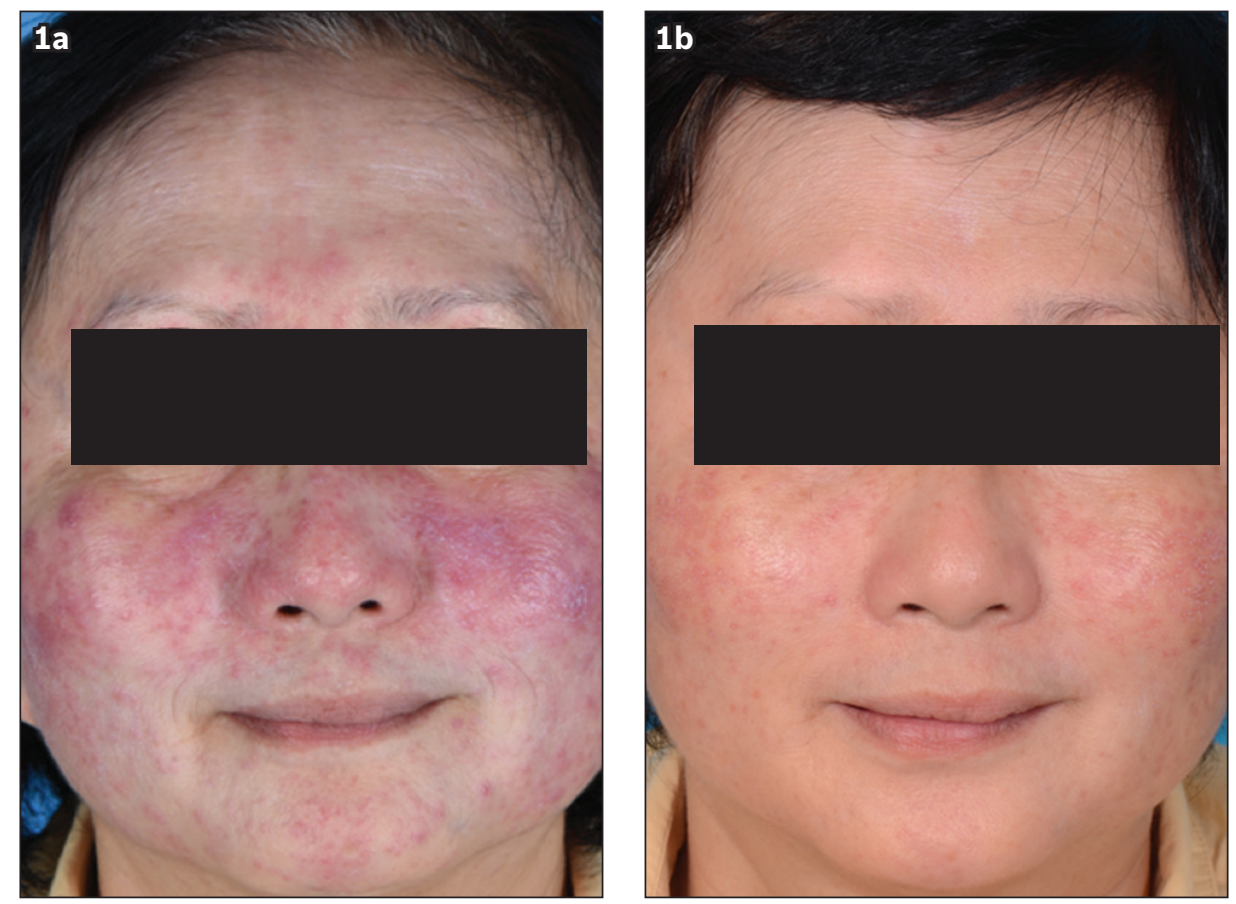

Fig. 1 Photographs show (a) numerous erythematous papulopustules and nodules on an erythematous base over the cheeks and chin of our patient; and (b) interval resolution with residual erythema over the cheeks after four months of treatment. 
In conclusion, this was a case of GR that responded to oral doxycycline, systemic steroids and topical brimonidine gel. It is important for clinicians to be aware of this condition and the mimics, as the abrupt onset and severely disfiguring presentation is a concern to patients. Histological confirmation should be considered in patients who do not respond to initial treatment.

\section{Yours sincerely,}

Wai Leong $\underline{K o k}^{1}$, Hazel H $\underline{\text { Oon }}^{1}$, Yoke Chin $\underline{\text { Giam}}^{1}$

${ }^{1}$ National Skin Centre, Singapore. kokwaileong@gmail.com

\section{REFERENCES}

1. Wilkin J, Dahl M, Detmar M, et al; National Rosacea Society Expert Committee. Standard grading system for rosacea: report of the National Rosacea Society Expert Committee on the classification and staging of rosacea. J Am Acad Dermatol 2002; 50:907-12.

2. Cribier B. Rosacea under the microscope: characteristic histological findings. J Eur Acad Dermatol Venereol 2013; 27:1336-43

3. Rocas D, Kanitakis J. Lupus miliaris disseminates faciei: report of a new case and brief literature review. Dermatol Online J 2013; 19:4.

4. Mihan R, Ayres S Jr. Perioral dermatitis. Arch Dermatol 1964; 89:803-5.

5. Lee GL, Zirwas MJ. Granulomatous rosacea and periorificial dermatitis: controversies and review of management and treatment. Dermatol Clin 2015; 33:447-55.

6. Odom R, Dahl M, Dover J, et al; National Rosacea Society Expert Committee on the Classification and Staging of Rosacea. Standard management options for rosacea, part 1: overview and broad spectrum of care. Cutis 2009; 84:43-7. 